\title{
Khatri-Rao Products for Operator Matrices Acting on the Direct Sum of Hilbert Spaces
}

\author{
Arnon Ploymukda and Pattrawut Chansangiam \\ Department of Mathematics, Faculty of Science, King Mongkut's Institute of Technology Ladkrabang, Chalongkrung Rd., \\ Bangkok 10520, Thailand
}

Correspondence should be addressed to Pattrawut Chansangiam; pattrawut.ch@kmitl.ac.th

Received 2 July 2016; Revised 20 September 2016; Accepted 18 October 2016

Academic Editor: Ralf Meyer

Copyright (C) 2016 A. Ploymukda and P. Chansangiam. This is an open access article distributed under the Creative Commons Attribution License, which permits unrestricted use, distribution, and reproduction in any medium, provided the original work is properly cited.

\begin{abstract}
We introduce the notion of Khatri-Rao product for operator matrices acting on the direct sum of Hilbert spaces. This notion generalizes the tensor product and Hadamard product of operators and the Khatri-Rao product of matrices. We investigate algebraic properties, positivity, and monotonicity of the Khatri-Rao product. Moreover, there is a unital positive linear map taking TracySingh products to Khatri-Rao products via an isometry.
\end{abstract}

\section{Introduction}

In matrix theory, there are various matrix products which are of interest in both theory and applications, such as the Kronecker product, Hadamard product, and Khatri-Rao product; see, for example, [1-3]. Denote by $M_{m, n}(\mathbb{C})$ the set of $m$-by- $n$ complex matrices and abbreviate $M_{n, n}(\mathbb{C})$ to $M_{n}(\mathbb{C})$. Recall that the Kronecker product of $A=\left[a_{i j}\right] \in M_{m, n}(\mathbb{C})$ and $B \in M_{p, q}(\mathbb{C})$ is given by

$$
A \widehat{\otimes} B=\left[a_{i j} B\right]_{i j} \in M_{m p, n q}(\mathbb{C}) .
$$

The Hadamard product of $A, B \in M_{m, n}(\mathbb{C})$ is defined by the entrywise product

$$
A \widehat{\odot} B=\left[a_{i j} b_{i j}\right] \in M_{m, n}(\mathbb{C}) .
$$

Now, let $A$ and $B$ be complex matrices partitioned into blocks $A_{i j}$ and $B_{i j}$ for each $i, j$ (the sizes of $A_{i j}$ and $B_{i j}$ may be different). Then, the Khatri-Rao product [4] of $A$ and $B$ is defined by

$$
A \widehat{\widehat{a}} B=\left[A_{i j} \widehat{\otimes} B_{i j}\right]_{i j} .
$$

When $A$ and $B$ are nonpartitioned (i.e., each has only one block), their Khatri-Rao product is just their Kronecker product. If $A$ and $B$ are entrywise partitioned (i.e., each block is a $1 \times 1$ matrix), then their Khatri-Rao product is their Hadamard product. Interesting algebraic, order, and analytic properties of this product were studied in the literature; see, for example, [5-12]. Their applications in statistics, computer science, and related fields can be seen, for example, in $[13,14]$.

The tensor product of Hilbert space operators is a natural extension of the Kronecker product to the infinitedimensional setting. Let $\mathbb{U}, \mathbb{U}^{\prime}, \mathbb{K}$, and $\mathbb{K}^{\prime}$ be Hilbert spaces. Recall that the tensor product of two operators $A: \mathbb{U} \rightarrow \mathbb{U}^{\prime}$ and $B: \mathbb{K} \rightarrow \mathbb{K}^{\prime}$ is the unique bounded linear operator from $\mathbb{U} \otimes \mathbb{K}$ into $\mathbb{H}^{\prime} \otimes \mathbb{K}^{\prime}$ such that, for all $x \in \mathbb{U}$ and $y \in \mathbb{K}$,

$$
(A \otimes B)(x \otimes y)=A x \otimes B y .
$$

In this paper, we generalize the tensor product of operators to the Khatri-Rao product of operator matrices acting on a direct sum of Hilbert spaces. We investigate fundamental properties of this operator product. Algebraically, this product is compatible with the addition, the scalar multiplication, the adjoint operation, and the direct sum of operators. By introducing suitable operator matrices, we can prove that there is a unital positive linear map taking the Tracy-Singh product $A \otimes B$ to the Khatri-Rao product $A \backsim B$. Hence, the Khatri-Rao product can be viewed as a generalization of the Hadamard product of operators. Moreover, positivity, strict 
positivity, and operator orderings are preserved under the Khatri-Rao product. Our result extends well-known results for Khatri-Rao products of complex matrices (see [4, 9, 15, 16]).

This paper is organized as follows. In Section 2, we provide some preliminaries about Tracy-Singh products for operators. These facts will be used in Sections 4 and 5. In Section 3, we introduce the Khatri-Rao product for operator matrices and deduce its algebraic properties. Section 4 explains how the Khatri-Rao product can be regarded as a generalization of the Hadamard product. Section 5 discusses positivity and monotonicity of Khatri-Rao products.

\section{Preliminaries on Tracy-Singh Products for Operators}

Throughout, let $\mathbb{H}$, $\mathbb{H}^{\prime}, \mathbb{K}$, and $\mathbb{K}^{\prime}$ be complex separable Hilbert spaces. When $\mathbb{X}$ and $\mathbb{Y}$ are Hilbert spaces, denote by $\mathscr{B}(\mathbb{X}, \mathbb{Y})$ the Banach space of bounded linear operators from $\mathbb{X}$ into $\mathbb{Y}$, and abbreviate $\mathscr{B}(\mathbb{X}, \mathbb{X})$ to $\mathscr{B}(\mathbb{X})$. If an operator $A \in \mathscr{B}(\mathbb{M})$ satisfies $\langle A x, x\rangle>0$, we write $A>0$. For selfadjoint operators $A, B \in \mathscr{B}(\mathbb{H})$, we write $A \geqslant B$ to mean that $A-B$ is a positive operator, while $A>B$ means that $A-B>0$.

Decompose

$$
\begin{aligned}
\mathbb{H} & =\bigoplus_{j=1}^{n} \mathbb{H}_{j}, \\
\mathbb{H}^{\prime} & =\bigoplus_{i=1}^{m} \mathbb{M}_{i}^{\prime}, \\
\mathbb{K} & =\bigoplus_{l=1}^{q} \mathbb{K}_{l}, \\
\mathbb{K}^{\prime} & =\bigoplus_{k=1}^{p} \mathbb{K}_{k}^{\prime},
\end{aligned}
$$

where all $\mathbb{H}_{j}, \mathbb{T}_{i}^{\prime}, \mathbb{K}_{l}$, and $\mathbb{K}_{k}^{\prime}$ are Hilbert spaces. For each $j$, $l$, let $M_{j}: \mathbb{H}_{j} \rightarrow \mathbb{H}$ and $N_{l}: \mathbb{K}_{l} \rightarrow \mathbb{K}$ be the canonical embeddings. For each $i$ and $k$, let $P_{i}: \mathbb{H}^{\prime} \rightarrow \mathbb{H}_{i}^{\prime}$ and $Q_{k}:$ $\mathbb{K}^{\prime} \rightarrow \mathbb{K}_{k}^{\prime}$ be the orthogonal projections. Two operators $A \in$ $\mathscr{B}\left(\mathbb{H}, \mathbb{H}^{\prime}\right)$ and $B \in \mathscr{B}\left(\mathbb{K}, \mathbb{K}^{\prime}\right)$ can thus be represented uniquely as operator matrices

$$
\begin{aligned}
& A=\left[A_{i j}\right]_{i, j=1}^{m, n}, \\
& B=\left[B_{k l}\right]_{k, l=1}^{p, q},
\end{aligned}
$$

where $A_{i j}=P_{i} A M_{j} \in \mathscr{B}\left(\mathbb{H}_{j}, \mathbb{H}_{i}^{\prime}\right)$ and $B_{k l}=Q_{k} B N_{l} \in$ $\mathscr{B}\left(\mathbb{K}_{l}, \mathbb{K}_{k}^{\prime}\right)$ for each $i, j, k$, and $l$. We define the Tracy-Singh product of $A$ and $B$ to be the bounded linear operator from $\bigoplus_{j, l=1}^{n, q} \mathbb{M}_{j} \otimes \mathbb{K}_{l}$ to $\bigoplus_{i, k=1}^{m, p} \mathbb{H}_{i}^{\prime} \otimes \mathbb{K}_{k}^{\prime}$ expressed in a block-matrix form

$$
A \otimes B=\left[\left[A_{i j} \otimes B_{k l}\right]_{k l}\right]_{i j} .
$$

Basic properties of the Tracy-Singh product are listed below.
Lemma 1. The Tracy-Singh product $(A, B) \mapsto A \otimes B$ is a bilinear map for operators. It is positive in the sense that if $A \geqslant 0$ and $B \geqslant 0$, then $A \otimes B \geqslant 0$.

\section{Compatibility of Khatri-Rao Products with Algebraic Operations}

In this section, we define the Khatri-Rao product for operator matrices and show that this product is compatible with certain algebraic operations of operators.

From now on, fix the following orthogonal decompositions of Hilbert spaces:

$$
\begin{aligned}
\mathbb{W} & =\bigoplus_{j=1}^{n} \mathbb{W}_{j}, \\
\mathbb{H}^{\prime} & =\bigoplus_{i=1}^{m} \mathbb{W}_{i}^{\prime}, \\
\mathbb{K} & =\bigoplus_{j=1}^{n} \mathbb{K}_{j}, \\
\mathbb{K}^{\prime} & =\bigoplus_{i=1}^{m} \mathbb{K}_{i}^{\prime} .
\end{aligned}
$$

That is, we fix how to partition any operator matrix in $\mathscr{B}\left(\mathbb{H}, \mathbb{U}^{\prime}\right)$ and $\mathscr{B}\left(\mathbb{K}, \mathbb{K}^{\prime}\right)$. We now extend the Khatri-Rao product of matrices [4] to that of operators on a Hilbert space.

Definition 2. Let $A=\left[A_{i j}\right]_{i, j=1}^{m, n} \in \mathscr{B}\left(\mathbb{H}, \mathbb{H}^{\prime}\right)$ and $B=$ $\left[B_{i j}\right]_{i, j=1}^{m, n} \in \mathscr{B}\left(\mathbb{K}, \mathbb{K}^{\prime}\right)$ be operators partitioned into matrices according to decomposition (8). We define the Khatri-Rao product of $A$ and $B$ to be the bounded linear operator from $\bigoplus_{j=1}^{n} \mathbb{H}_{j} \otimes \mathbb{K}_{j}$ to $\bigoplus_{i=1}^{m} \mathbb{W}_{i}^{\prime} \otimes \mathbb{K}_{i}^{\prime}$ represented by the block-matrix

$$
A \boxminus B=\left[A_{i j} \otimes B_{i j}\right]_{i, j=1}^{m, n} .
$$

If both $A$ and $B$ are $1 \times 1$ block operator matrices, then $A \otimes B$ is $A \otimes B$. When $\mathbb{T}_{i}=\mathbb{K}_{i}=\mathbb{C}$ and $\mathbb{H}_{j}^{\prime}=\mathbb{K}_{j}^{\prime}=\mathbb{C}$ for all $i, j$, the Khatri-Rao product is the Hadamard product of complex matrices.

Next, we shall show that the Khatri-Rao product of two linear maps induced by matrices is just the linear map induced by the Khatri-Rao product of these matrices. Recall that, for each $A \in M_{m, n}(\mathbb{C})$ and $B \in M_{p, q}(\mathbb{C})$, the induced maps,

$$
\begin{aligned}
L_{A}: \mathbb{C}^{n} \longrightarrow \mathbb{C}^{m}, \quad x \longmapsto A x, \\
L_{B}: \mathbb{C}^{q} \longrightarrow \mathbb{C}^{p}, \quad y \longmapsto B y,
\end{aligned}
$$

are bounded linear operators. We identify $\mathbb{C}^{n} \otimes \mathbb{C}^{q}$ with $\mathbb{C}^{n q}$ together with the canonical bilinear map $(x, y) \mapsto x \widehat{\otimes} y$ for each $(x, y) \in \mathbb{C}^{n} \times \mathbb{C}^{q}$.

Lemma 3. For any $A \in M_{m, n}(\mathbb{C})$ and $B \in M_{p, q}(\mathbb{C})$, one has

$$
L_{A} \otimes L_{B}=L_{A \hat{\otimes} B} .
$$


Proof. Recall that the Kronecker product of matrices has the following property (see, e.g., [3]):

$$
(A \widehat{\otimes} B)(C \widehat{\otimes} D)=A C \widehat{\otimes} B D
$$

provided that all matrix products are well defined. It follows that, for any $x \in \mathbb{C}^{n}$ and $y \in \mathbb{C}^{q}$,

$$
\begin{aligned}
\left(L_{A} \otimes L_{B}\right)(x \otimes y) & =L_{A}(x) \otimes L_{B}(y) \\
& =L_{A}(x) \widehat{\otimes} L_{B}(y)=A x \widehat{\otimes} B y \\
& =(A \widehat{\otimes} B)(x \widehat{\otimes} y)=(A \widehat{\otimes} B)(x \otimes y) \\
& =L_{A \widehat{\otimes} B}(x \otimes y) .
\end{aligned}
$$

The uniqueness of tensor products implies that $L_{A} \otimes L_{B}=$ $L_{A \widehat{\otimes} B}$.

Proposition 4. For any complex matrices $A=\left[A_{i j}\right]$ and $B=$ $\left[B_{i j}\right]$ partitioned in block-matrix form, one has

$$
L_{A} \unrhd L_{B}=L_{A \widehat{\ominus} B} .
$$

Proof. Recall that the $(i, j)$ th block of the matrix representation of $L_{A}$ is $L_{A_{i j}}$. By Lemma 3, we obtain $L_{A} \bullet L_{B}=$ $\left[L_{A_{i j}} \otimes L_{B_{i j}}\right]_{i j}=\left[L_{A_{i j} \hat{\otimes} B_{i j}}\right]_{i j}=L_{A \widehat{\ominus} B}$.

The next result states that the Khatri-Rao product is bilinear and compatible with the adjoint operation.

Proposition 5. Let $A \in \mathscr{B}\left(\mathbb{H}, \mathbb{H}^{\prime}\right)$ and $B, C \in \mathscr{B}\left(\mathbb{K}, \mathbb{K}^{\prime}\right)$ be operator matrices, and let $\alpha \in \mathbb{C}$. Then,

$$
\begin{aligned}
(A \boxminus B)^{*} & =A^{*} \boxminus B^{*}, \\
A \boxminus(B+C) & =A \boxminus B+A \boxminus C, \\
(B+C) \boxminus A & =B \boxminus A+C \boxminus A, \\
(\alpha A) \boxminus B & =\alpha(A \boxminus B)=A \boxminus(\alpha B) .
\end{aligned}
$$

Proof. Since $A^{*}=\left[A_{j i}^{*}\right]_{i j}$ and $B^{*}=\left[B_{j i}^{*}\right]_{i j}$, we obtain

$$
\begin{aligned}
(A \boxminus B)^{*} & =\left[\left(A_{i j} \otimes B_{i j}\right)^{*}\right]_{i j}=\left[A_{j i}^{*} \otimes B_{j i}^{*}\right]_{i j} \\
& =A^{*} \bullet B^{*} .
\end{aligned}
$$

The fact that $(B+C)_{i j}=B_{i j}+C_{i j}$ for all $i, j$ together with the left distributivity of the tensor product over the addition implies

$$
\begin{aligned}
A \boxminus(B+C) & =\left[A_{i j} \otimes\left(B_{i j}+C_{i j}\right)\right]_{i j} \\
& =\left[\left(A_{i j} \otimes B_{i j}\right)+\left(A_{i j} \otimes C_{i j}\right)\right]_{i j} \\
& =A \boxminus B+A \boxminus C .
\end{aligned}
$$

Similarly, we obtain property (17). Since $(\alpha A)_{i j}=\alpha A_{i j}$ for all $i, j$, we get

$$
\begin{aligned}
(\alpha A) \boxminus B & =\left[\left(\alpha A_{i j}\right) \otimes B_{i j}\right]_{i j}=\left[\alpha\left(A_{i j} \boxminus B_{i j}\right)\right]_{i j} \\
& =\alpha(A \odot B) .
\end{aligned}
$$

Similarly, $A \boxminus(\alpha B)=\alpha(A \boxminus B)$.

By property (15), the self-adjointness of operators is closed under taking Khatri-Rao products; that is, if $A$ and $B$ are self-adjoint, then so is $A \bowtie B$. The next proposition shows that, in order to compute the Khatri-Rao product of operator matrices, we can freely merge the partition of each operator.

Proposition 6. Let $A=\left[A_{i j}\right]_{i, j=1}^{m, n} \in \mathscr{B}\left(\mathbb{H}, \mathbb{H}^{\prime}\right)$ and $B$ $=\left[B_{i j}\right]_{i, j=1}^{m, n} \in \mathscr{B}\left(\mathbb{K}, \mathbb{K}^{\prime}\right)$ be operator matrices represented according to decomposition (8). We merge the partition of $A$ to be $A=\left[A^{k l}\right]_{k, l=1}^{r, s}$, where $r$, s are given natural numbers such that $r \leqslant m$ and $s \leqslant n$. Here, each operator $A^{k l}$ is of $m_{k} \times n_{l}$ block in which the $(g, h)$ th block of $A^{k l}$ is the $(u, v)$ th block of A, where

$$
u= \begin{cases}g, & k=1 \\ \sum_{i=1}^{k-1} m_{i}+g, & k>1\end{cases}
$$$$
\sum_{k=1}^{r} m_{k}=m,
$$

Similarly, we repartition $B=\left[B^{k l}\right]_{k, l=1}^{r, s}$, where each operator $B^{k l}$ is of $m_{k} \times n_{l}$ block in which the $(g, h)$ th block of $B^{k l}$ is the $(u, v)$ th block of $B$. Then,

$$
\begin{aligned}
A \boxminus B & =\left[A^{k l} \boxminus B^{k l}\right]_{k l} \\
& =\left[\begin{array}{ccc}
A^{11} \bullet B^{11} & \ldots & A^{1 s} \bullet B^{1 s} \\
\vdots & \ddots & \vdots \\
A^{r 1} \bullet B^{r 1} & \cdots & A^{r s} \bullet B^{r s}
\end{array}\right] .
\end{aligned}
$$

That is, each $(k, l)$ th block of $A \boxminus B$ is just $A^{k l} \boxminus B^{k l}$. 
Proof. Write $A \boxminus B=\left[C^{k l}\right]_{k, l=1}^{r, s}$, where $C^{k l}$ is $m_{k} \times n_{l}$ block operator matrix such that the $(g, h)$ th block of $C^{k l}$ is the $(u, v)$ th block of $A \square B$. We know that the $(u, v)$ th block of $A \boxminus B$ is $A_{u v} \otimes B_{u v}$. Then,

$$
\begin{aligned}
C^{11} & =\left[\begin{array}{ccc}
A_{11} \otimes B_{11} & \cdots & A_{1 n_{1}} \otimes B_{1 n_{1}} \\
\vdots & \ddots & \vdots \\
A_{m_{1} 1} \otimes B_{m_{1} 1} & \cdots & A_{m_{1} n_{1}} \otimes B_{m_{1} n_{1}}
\end{array}\right] \\
& =\left[\begin{array}{ccc}
A_{11} & \cdots & A_{1 n_{1}} \\
\vdots & \ddots & \vdots \\
A_{m_{1} 1} & \cdots & A_{m_{1} n_{1}}
\end{array}\right] \bullet\left[\begin{array}{ccc}
B_{11} & \cdots & B_{1 n_{1}} \\
\vdots & \ddots & \vdots \\
B_{m_{1} 1} & \cdots & B_{m_{1} n_{1}}
\end{array}\right] \\
= & A^{11} \odot B^{11} .
\end{aligned}
$$

Similarly, we have $C^{k l}=A^{k l} \square B^{k l}$ for all $k=1, \ldots, r$ and $l=1, \ldots, s$.

Recall that the direct sum of $A_{i} \in \mathscr{B}\left(\mathbb{U}_{i}, \mathbb{H}_{i}^{\prime}\right), i=1, \ldots, n$, is defined to be the operator matrix

$$
A_{1} \oplus \cdots \oplus A_{n}=\left[\begin{array}{cccc}
A_{1} & 0 & \cdots & 0 \\
0 & A_{2} & \cdots & 0 \\
\vdots & \vdots & \ddots & \vdots \\
0 & 0 & \cdots & A_{n}
\end{array}\right] .
$$

The next result shows that the Khatri-Rao product is compatible with the direct sum of operators.

Proposition 7. For each $i=1, \ldots, n$, let $A_{i} \in \mathscr{B}\left(\mathbb{H}_{i}, \mathbb{H}_{i}^{\prime}\right)$ and $B_{i} \in \mathscr{B}\left(\mathbb{K}_{i}, \mathbb{K}_{i}^{\prime}\right)$ be compatible operator matrices. Then,

$$
\left(\bigoplus_{i=1}^{n} A_{i}\right) \cdot\left(\bigoplus_{i=1}^{n} B_{i}\right)=\bigoplus_{i=1}^{n}\left(A_{i} \boxminus B_{i}\right) .
$$

Proof. It follows directly from Proposition 6.

In summary, the Khatri-Rao product is compatible with fundamental algebraic operations for operators.

\section{The Khatri-Rao Product as a Generalization of the Hadamard Product}

In this section, we explain how the Khatri-Rao product can be viewed as a generalization of the Hadamard product. To do this, we construct two isometries which identify which blocks of the Tracy-Singh product we need to get the KhatriRao product.

Fix a countable orthonormal basis $\mathscr{E}$ for $\mathbb{\sharp}$. Recall that the Hadamard product of $A$ and $B$ in $\mathscr{B}(\mathbb{Q})$ is defined to be the operator $A \odot B$ in $\mathscr{B}(\mathbb{Q})$ such that

$$
\langle(A \odot B) e, e\rangle=\langle A e, e\rangle\langle B e, e\rangle
$$

for all $e \in \mathscr{E}$. More explicitly, it was shown in [15] that

$$
A \odot B=U^{*}(A \otimes B) U,
$$

where $U: \mathbb{U} \rightarrow \mathbb{U} \otimes \mathbb{W}$ is the isometry defined by $U e=e \otimes e$ for all $e \in \mathscr{E}$. When $\mathbb{H}=\mathbb{C}^{n}$ and $\mathscr{E}$ is the standard ordered basis of $\mathbb{C}^{n}$, the Hadamard product of two matrices reduces to the entrywise product (2).

We now extend selection matrices in [9] to selection operators. Fix an ordered 4-tuple $\left(\mathbb{\square}, \mathbb{U}^{\prime}, \mathbb{K}, \mathbb{K}^{\prime}\right)$ of Hilbert spaces endowed with decomposition (8). For each $r=$ $1, \ldots, m$, consider the operator matrix

$$
E_{r}=\left[E_{g h}^{(r)}\right]_{g, h=1}^{m, m}: \bigoplus_{i=1}^{m} \mathbb{W}_{i}^{\prime} \otimes \mathbb{K}_{i}^{\prime} \longrightarrow \bigoplus_{i=1}^{m} \mathbb{W}_{r}^{\prime} \otimes \mathbb{K}_{i}^{\prime},
$$

where $E_{g h}^{(r)}$ is the identity operator if $g=h=r$ and the others are zero operators. Similarly, for $s=1, \ldots, n$, we define the operator matrix

$$
F_{s}=\left[F_{g h}^{(s)}\right]_{g, h=1}^{n, n}: \bigoplus_{j=1}^{n} \mathbb{H}_{j} \otimes \mathbb{K}_{j} \longrightarrow \bigoplus_{j=1}^{n} \mathbb{H}_{s} \otimes \mathbb{K}_{j},
$$

where $F_{g h}^{(s)}$ is the identity operator if $g=h=s$ and the others are zero operators. Now, construct

$$
\begin{aligned}
& Z_{1}=\left[\begin{array}{c}
E_{1} \\
\vdots \\
E_{m}
\end{array}\right], \\
& Z_{2}=\left[\begin{array}{c}
F_{1} \\
\vdots \\
F_{n}
\end{array}\right] .
\end{aligned}
$$

We call $Z_{1}$ and $Z_{2}$ selection operators associated with the ordered tuple $\left(\mathbb{W}, \mathbb{U}^{\prime}, \mathbb{K}, \mathbb{K}^{\prime}\right)$. Notice that $Z_{1}$ depends only on the ordered tuple $\left(\mathbb{H}^{\prime}, \mathbb{K}^{\prime}\right)$ and how we decomposed $\mathbb{\mathbb { N } ^ { \prime }}$ and $\mathbb{K}^{\prime}$. The operator $Z_{2}$ depends on $(\mathbb{H}, \mathbb{K})$ and how we decomposed $\mathbb{n}$ and $\mathbb{K}$. For instance, an ordered tuple $\left(\mathbb{U}, \mathbb{U}^{\prime}, \mathbb{K}, \mathbb{K}^{\prime}\right)$ with decompositions

$$
\begin{aligned}
\mathbb{W} & =\mathbb{H}_{1} \oplus \mathbb{W}_{2} \oplus \mathbb{H}_{3}, \\
\mathbb{W} & =\mathbb{W}_{1}^{\prime} \oplus \mathbb{U}_{2}^{\prime}, \\
\mathbb{K} & =\mathbb{K}_{1} \oplus \mathbb{K}_{2} \oplus \mathbb{K}_{3}, \\
\mathbb{K}^{\prime} & =\mathbb{K}_{1}^{\prime} \oplus \mathbb{K}_{2}^{\prime}
\end{aligned}
$$

has the following selection operators:

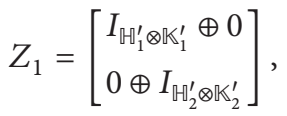

$$
\begin{aligned}
& Z_{2}=\left[\begin{array}{c}
I_{\mathbb{1}_{1} \otimes \mathbb{K}_{1}} \oplus 0 \oplus 0 \\
0 \oplus I_{\mathbb{I}_{2} \otimes \mathbb{K}_{2}} \oplus 0 \\
0 \oplus 0 \oplus I_{\mathbb{H}_{3} \otimes \mathbb{K}_{3}}
\end{array}\right] .
\end{aligned}
$$


In the case of $\mathbb{U}=\mathbb{M}^{\prime}$ and $\mathbb{K}=\mathbb{K}^{\prime}$, construction (31) gives

$$
Z_{1}=Z_{2}=Z \text {. }
$$

If $\left(Z_{1}, Z_{2}\right)$ is the ordered pair of selection operators associated with the ordered tuple $\left(\mathbb{H}, \mathbb{H}^{\prime}, \mathbb{K}, \mathbb{K}^{\prime}\right)$ with decompositions given by (8), then $\left(Z_{2}, Z_{1}\right)$ is the ordered pair of selection operators associated with the ordered collection $\left(\mathbb{M}^{\prime}, \mathbb{H}, \mathbb{K}^{\prime}, \mathbb{K}\right)$ with the same decompositions.

Lemma 8. Let $Z_{1}$ and $Z_{2}$ be selection operators defined by (31). Then, for $i=1,2$,

(i) $Z_{i}^{*} Z_{i}=I$; that is, $Z_{i}$ is an isometry;

(ii) $0 \leqslant Z_{i} Z_{i}^{*} \leqslant I$.

Proof. A direct computation shows that $Z_{1}^{*} Z_{1}=I$ and $Z_{2}^{*} Z_{2}=I$. We know that $E_{i} E_{i}^{*}$ is an $m \times m$ block operator matrix which consists only of zero and identity operators. More precisely, the $(i, i)$ th block of $E_{i} E_{i}^{*}$ is the identity operator and $E_{i} E_{j}^{*}=0$ for $i \neq j$. Then,

$$
\begin{aligned}
Z_{1} Z_{1}^{*}= & {\left[\begin{array}{cccc}
E_{1} E_{1}^{*} & E_{1} E_{2}^{*} & \cdots & E_{1} E_{m}^{*} \\
E_{2} E_{1}^{*} & E_{2} E_{2}^{*} & \cdots & E_{2} E_{m}^{*} \\
\vdots & \vdots & \ddots & \vdots \\
E_{m} E_{1}^{*} & E_{m} E_{2}^{*} & \cdots & E_{m} E_{m}^{*}
\end{array}\right] } \\
= & {\left[\begin{array}{cccc}
E_{1} E_{1}^{*} & 0 & \cdots & 0 \\
0 & E_{2} E_{2}^{*} & \cdots & 0 \\
\vdots & \vdots & \ddots & \vdots \\
0 & 0 & \cdots & E_{m} E_{m}^{*}
\end{array}\right] . }
\end{aligned}
$$

Since $E_{i} E_{i}^{*} \leqslant I$ for all $i=1, \ldots, m$, we have $Z_{1} Z_{1}^{*} \leqslant I$. Similarly, $Z_{2} Z_{2}^{*} \leqslant I$.

Next, we relate the Khatri-Rao and the Tracy-Singh product of operators.

Theorem 9. For any operator matrices $A \in \mathscr{B}\left(\mathbb{M}, \mathbb{M}^{\prime}\right)$ and $B \in$ $\mathscr{B}\left(\mathbb{K}, \mathbb{K}^{\prime}\right)$, one has

$$
A \boxminus B=Z_{1}^{*}(A \otimes B) Z_{2},
$$

where $Z_{1}$ and $Z_{2}$ are the selection operators defined by (31). If $\mathbb{M}=\mathbb{M}^{\prime}$ and $\mathbb{K}=\mathbb{K}^{\prime}, A \in \mathscr{B}(\mathbb{M})$ and $B \in \mathscr{B}(\mathbb{K})$, then

$$
A \bowtie B=Z^{*}(A \otimes B) Z,
$$

where $Z$ is the selection operator defined by (34).

Proof. Let $B(i)$ denote the $i$ th column of $B$ for $i=1, \ldots, n$. Then, we have

$$
\begin{aligned}
& Z_{1}^{*}(A \otimes B) Z_{2} \\
& =\left[\begin{array}{lll}
E_{1}^{*} & \cdots & E_{m}^{*}
\end{array}\right]\left[\begin{array}{ccc}
A_{11} \otimes B & \cdots & A_{1 n} \otimes B \\
\vdots & \ddots & \vdots \\
A_{m 1} \otimes B & \cdots & A_{m n} \otimes B
\end{array}\right]\left[\begin{array}{c}
F_{1} \\
\vdots \\
F_{n}
\end{array}\right]
\end{aligned}
$$

$$
\begin{gathered}
=\left[\begin{array}{lll}
E_{1}^{*} & \cdots & E_{m}^{*}
\end{array}\right]\left[\begin{array}{c}
\left(A_{11} \otimes B\right) F_{1}+\cdots+\left(A_{1 n} \otimes B\right) F_{n} \\
\left(A_{m 1} \otimes B\right) F_{1}+\cdots+\left(A_{m n} \otimes B\right) F_{n}
\end{array}\right] \\
=\left[\begin{array}{lll}
E_{1}^{*} & \cdots & E_{m}^{*}
\end{array}\right]\left[\begin{array}{ccc}
A_{11} \otimes B(1) & \cdots & A_{1 n} \otimes B(n) \\
\vdots & \\
A_{m 1} \otimes B(1) & \cdots & A_{m n} \otimes B(n)
\end{array}\right] \\
=\left[\begin{array}{ccc}
A_{11} \otimes B_{11} & \cdots & A_{1 n} \otimes B_{1 n} \\
\vdots & \ddots & \vdots \\
A_{m 1} \otimes B_{m 1} & \cdots & A_{m n} \otimes B_{m n}
\end{array}\right]=A \bowtie B .
\end{gathered}
$$

If $\mathbb{M}=\mathbb{M}^{\prime}$ and $\mathbb{K}=\mathbb{K}^{\prime}$, then $Z_{1}=Z_{2}$ and (36) becomes (37).

We mention that Theorem 9 is an extension of both [9, Theorem 3] and result (28) in [15].

Remark 10. If we partition $A$ and $B$ into row operator matrices, we have

$$
A \boxminus B=(A \otimes B) Z_{2} .
$$

If both $A$ and $B$ are column operator matrices, then

$$
A \boxminus B=Z_{1}^{*}(A \otimes B) .
$$

Comparing (28) and (37), Theorem 9 shows that the Khatri-Rao product can be regarded as a generalization of the Hadamard product.

Recall that a map $\Phi$ between two $C^{*}$-algebras is said to be positive if $\Phi$ preserves positive elements. The map $\Phi$ is unital if $\Phi$ preserves the multiplicative identity.

Corollary 11. There is a unital positive linear map

$$
\Phi: \mathscr{B}\left(\bigoplus_{i, j=1}^{n, n} \mathbb{H}_{i} \otimes \mathbb{K}_{j}\right) \longrightarrow \mathscr{B}\left(\bigoplus_{i=1}^{n} \mathbb{H}_{i} \otimes \mathbb{K}_{i}\right)
$$

such that $\Phi(A \otimes B)=A \boxminus B$ for any $A \in \mathscr{B}(\mathbb{M})$ and $B \in \mathscr{B}(\mathbb{K})$.

Proof. Define $\Phi(X)=Z^{*} X Z$, where $Z$ is the selection operator defined by (37) in Theorem 9. The map $\Phi$ is clearly linear and positive. The map $\Phi$ is unital since $Z$ is an isometry (Lemma 8).

Corollary 11 provides a natural way to derive operator inequalities concerning Khatri-Rao products from existing inequalities for Tracy-Singh products.

The next result extends [16, Corollary 3] to the case of Khatri-Rao and Tracy-Singh products of operators.

Corollary 12. Let $A=A_{1} \oplus \cdots \oplus A_{n}$ and $B=B_{1} \oplus \cdots \oplus B_{n}$ be operators in $\mathscr{B}(\mathbb{H})$ and $\mathscr{B}(\mathbb{K})$, respectively. Then,

$$
\begin{aligned}
Z^{*}(A \otimes B) & =(A \boxminus B) Z^{*}, \\
(A \otimes B) Z & =Z(A \boxminus B) .
\end{aligned}
$$


Proof. Using the fact that $E_{i} E_{i}^{*} X=X_{i}=X E_{i} E_{i}^{*}$ and $E_{i} E_{j}^{*} X=$ $0=X E_{i} E_{j}^{*}$ if $i \neq j$, where $X=X_{1} \oplus \cdots \oplus X_{n}$, we compute

$$
\begin{aligned}
& Z Z^{*}(A \otimes B) \\
& =\left[\begin{array}{ccc}
E_{1} E_{1}^{*} & \cdots & 0 \\
\vdots & \ddots & \vdots \\
0 & \cdots & E_{n} E_{n}^{*}
\end{array}\right]\left[\begin{array}{ccc}
A_{1} \otimes B & \cdots & 0 \\
\vdots & \ddots & \vdots \\
0 & \cdots & A_{n} \otimes B
\end{array}\right] \\
& =\left[\begin{array}{ccc}
E_{1} E_{1}^{*}\left(A_{1} \otimes B\right) & \cdots & 0 \\
\vdots & \ddots & \vdots \\
0 & \cdots & E_{n} E_{n}^{*}\left(A_{n} \otimes B\right)
\end{array}\right]
\end{aligned}
$$

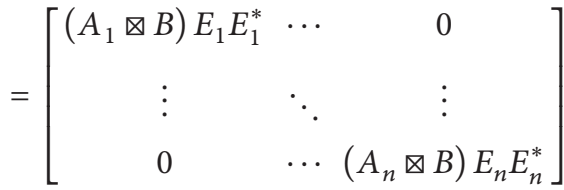

$$
\begin{aligned}
& =\left[\begin{array}{ccc}
A_{1} \otimes B & \cdots & 0 \\
\vdots & \ddots & \vdots \\
0 & \cdots & A_{n} \otimes B
\end{array}\right]\left[\begin{array}{ccc}
E_{1} E_{1}^{*} & \cdots & 0 \\
\vdots & \ddots & \vdots \\
0 & \cdots & E_{n} E_{n}^{*}
\end{array}\right] \\
& =(A \otimes B) Z Z^{*} .
\end{aligned}
$$

By applying Theorem 9, we get

$$
\begin{aligned}
Z^{*}(A \otimes B) & =Z^{*} Z Z^{*}(A \otimes B)=Z^{*}(A \otimes B) Z Z^{*} \\
& =(A \boxminus B) Z^{*} .
\end{aligned}
$$

Similarly, $(A \otimes B) Z=Z(A \boxminus B)$.

\section{Positivity and Monotonicity of Khatri-Rao Products}

In this section, we show that the Khatri-Rao product preserves positivity and strict positivity. It follows that operator orderings are preserved under Khatri-Rao products.

Theorem 13. Let $A \in \mathscr{B}(\mathbb{W})$ and $B \in \mathscr{B}(\mathbb{K})$ be operator matrices. If $A \geqslant 0$ and $B \geqslant 0$, then $A \boxminus B \geqslant 0$.

Proof. It follows from the positivity of the Tracy-Singh product (Lemma 1) and Theorem 9.

The next result provides the monotonicity of Khatri-Rao product which is an extension of [9, Theorem 5] to the case of operators.

Corollary 14. Let $A_{1}, A_{2} \in \mathscr{B}(\mathbb{M})$ and $B_{1}, B_{2} \in \mathscr{B}(\mathbb{K})$. If $A_{1} \geqslant A_{2} \geqslant 0$ and $B_{1} \geqslant B_{2} \geqslant 0$, then $A_{1} \square B_{1} \geqslant A_{2} \square B_{2}$.
Proof. Applying Proposition 5 and Theorem 13 yields

$$
\begin{aligned}
& \left(A_{1} \triangleright B_{1}\right)-\left(A_{2} \triangleright B_{2}\right) \\
& =A_{1} \boxminus B_{1}-A_{2} \boxminus B_{1}+A_{2} \boxminus B_{1}-A_{2} \boxminus B_{2} \\
& =\left(A_{1}-A_{2}\right) \boxminus B_{1}+A_{2} \boxminus\left(B_{1}-B_{2}\right) \geqslant 0 \text {. }
\end{aligned}
$$

Thus, $A_{1} \bowtie B_{1} \geqslant A_{2} \bowtie B_{2}$.

Now, we will develop the result of [9, Theorem 6] to the case of Khatri-Rao product of operators.

Theorem 15. Let $A \in \mathscr{B}(\mathbb{H})$ and $B \in \mathscr{B}(\mathbb{K})$ be operator matrices. If $A>0$ and $B>0$, then $A \square B>0$.

Proof. The strict positivity of $A$ and the spectral theorem imply the existence of an increasing sequence $\left(\mathbb{U}_{n}\right)_{n=1}^{\infty}$ of closed subspaces of $\mathbb{W}$ such that, for each $n \in \mathbb{N}$,

$$
\langle A x, x\rangle \geqslant \frac{1}{n}\|x\|^{2}
$$

for each $x \in \mathbb{H}_{n}$. Let $P_{n}$ be the orthogonal projection onto $\mathbb{U}_{n}$ for each $n \in \mathbb{N}$. There are similar subspaces $\mathbb{K}_{n}$ and orthogonal projections $Q_{n}$ for the operator $B$. Then, for each $n \in \mathbb{N}$, we have $A \geqslant(1 / n) P_{n}$ and $B \geqslant(1 / n) Q_{n}$ and hence

$$
A \boxminus B \geqslant \frac{1}{n^{2}} P_{n} \boxminus Q_{n}
$$

by Corollary 14 . Since the union of the subspaces $\mathbb{M}_{n}$ in $\mathbb{M}$ and of the subspaces $\mathbb{K}_{n}$ in $\mathbb{K}$ is dense, it follows that, for any $z \in$

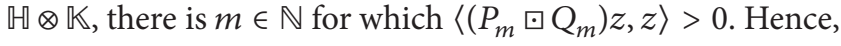

$$
\langle(A \boxminus B) z, z\rangle \geqslant \frac{1}{m^{2}}\left\langle\left(P_{m} \boxminus Q_{m}\right) z, z\right\rangle>0 .
$$

This shows that $A \boxminus B>0$.

Corollary 16. Let $A_{1}, A_{2} \in \mathscr{B}(\mathbb{H})$ and $B_{1}, B_{2} \in \mathscr{B}(\mathbb{K})$. If $A_{1}>A_{2}>0$ and $B_{1}>B_{2}>0$, then $A_{1} \boxminus B_{1}>A_{2} \vee B_{2}$.

Proof. The proof is similar to that of Corollary 14. Instead of Theorem 13, we apply Theorem 15.

Finally, we mention that, by using the results in this paper, we can develop further operator identities/inequalities parallel to matrix results for Khatri-Rao products.

\section{Competing Interests}

The authors declare that they have no competing interests.

\section{Acknowledgments}

This work was supported by the Thailand Research Fund. The second author would like to thank the Thailand Research Fund for the financial support. 


\section{References}

[1] S. Liu and G. Trenkler, "Hadamard, Khatri-Rao and other matrix products," International Journal of Computer and System Sciences, vol. 4, pp. 160-177, 2008.

[2] C. F. Van Loan, "The ubiquitous Kronecker product," Journal of Computational and Applied Mathematics, vol. 123, no. 1-2, pp. 85-100, 2000.

[3] H. Zhang and F. Ding, "On the Kronecker products and their applications," Journal of Applied Mathematics, vol. 2013, Article ID 296185, 8 pages, 2013.

[4] C. G. Khatri and C. R. Rao, "Solutions to some functional equations and their applications to characterization of probability distributions," Sankhya, vol. 30, pp. 167-180, 1968.

[5] Z. A. Al Zhour and A. Kilicman, "Extension and generalization inequalities involving the Khatri-Rao product of several positive matrices," Journal of Inequalities and Applications, vol. 2006, Article ID 80878, 21 pages, 2006.

[6] C.-G. Cao, X. Zhang, and Z.-P. Yang, "Some inequalities for the Khatri-Rao product of matrices," Electronic Journal of Linear Algebra, vol. 9, pp. 276-281, 2002.

[7] H. Civciv and R. Taurkmen, "On the bounds for $l^{p}$ norms of Khatri-Rao and Tracy-Singh products of Cauchy-Toeplitz matrices," Selcuk Journal of Applied Mathematics, vol. 6, no. 2, pp. 43-52, 2005.

[8] X. X. Feng and Z. P. Yang, "Lowner partial ordering inequalities on the Khatri-Rao product of matrices," Gongcheng Shuxue Xuebao, vol. 19, pp. 106-110, 2002.

[9] S. Liu, "Matrix results on the Khatri-Rao and Tracy-Singh products," Linear Algebra and Its Applications, vol. 289, no. 1-3, pp. 267-277, 1999.

[10] S. Liu, "Several inequalities involving Khatri-Rao products of positive semidefinite matrices," Linear Algebra and Its Applications, vol. 354, pp. 175-186, 2002.

[11] Z.-P. Yang, X. Zhang, and C.-G. Cao, "Inequalities involving Khatri-Rao products of Hermitian matrices," The Korean Journal of Computational \& Applied Mathematics, vol. 9, no. 1, pp. 125-133, 2002.

[12] X. Zhang, Z.-P. Yang, and C.-G. Cao, "Matrix inequalities involving the Khatri-Rao product," Archivum Mathematicum, vol. 38, no. 4, pp. 265-272, 2002.

[13] S. Liu, Contributions to Matrix Calculus and Applications in Econometrics, Tinbergen Institute Research Series no. 106, Thesis Publishers, Amsterdam, The Netherlands, 1995.

[14] C. R. Rao and M. B. Rao, Matrix Algebra and Its Applications to Statistics and Econometrics, World Scientific, Singapore, 1998.

[15] J. I. Fujii, "The Marcus-Khan theorem for Hilbert space operators," Mathematica Japonica, vol. 41, no. 3, pp. 531-535, 1995.

[16] G. Visick, "A quantitative version of the observation that the Hadamard product is a principal submatrix of the Kronecker product," Linear Algebra and Its Applications, vol. 304, no. 1-3, pp. 45-68, 2000. 


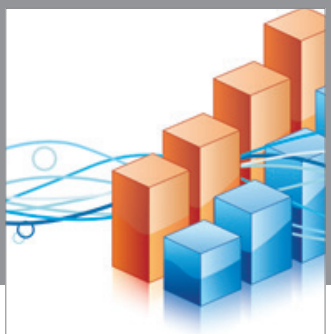

Advances in

Operations Research

vatem alat4

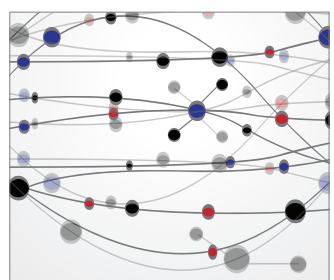

\section{The Scientific} World Journal
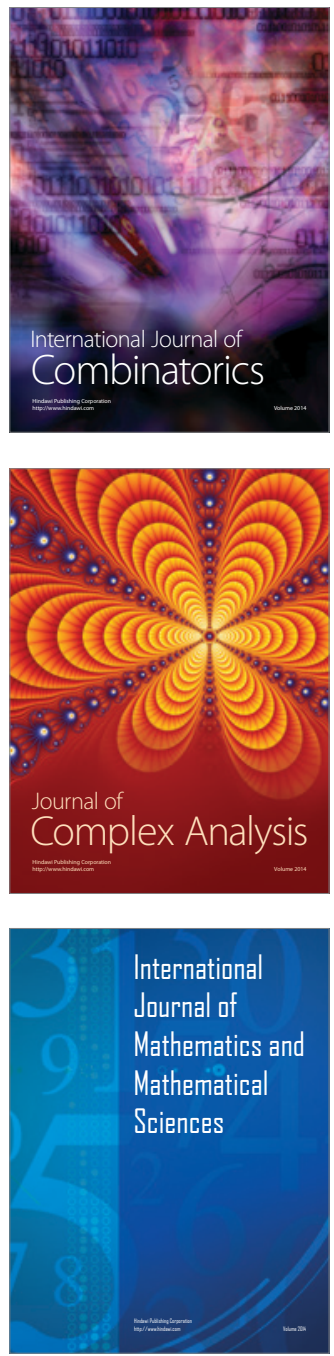
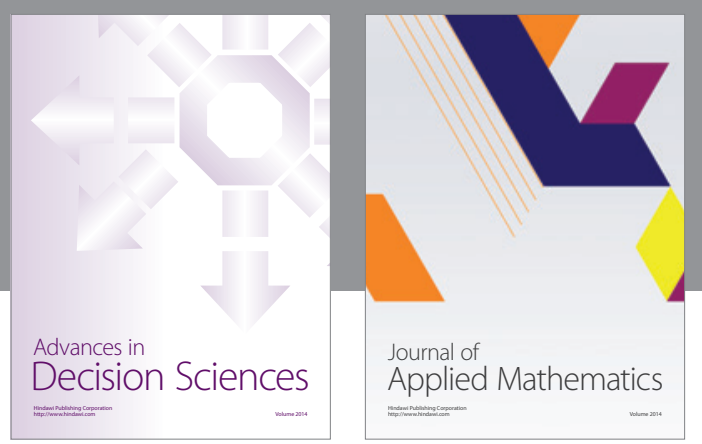

Algebra

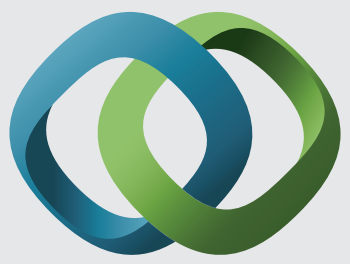

\section{Hindawi}

Submit your manuscripts at

http://www.hindawi.com
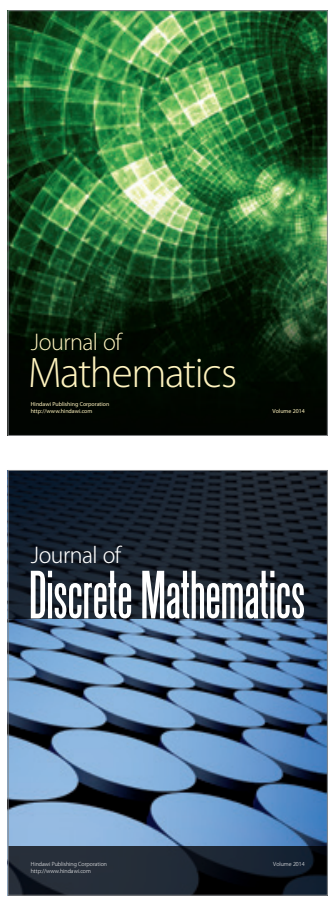

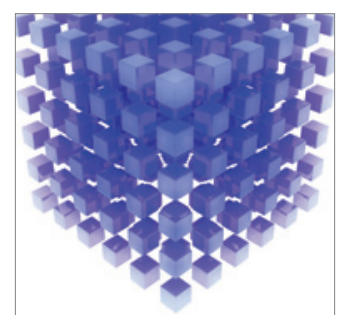

Mathematical Problems in Engineering
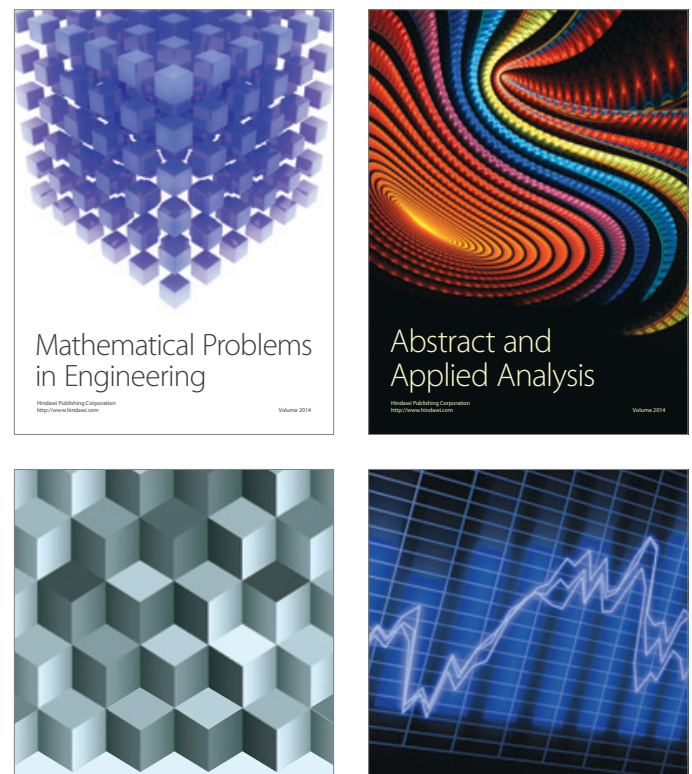

Journal of

Function Spaces

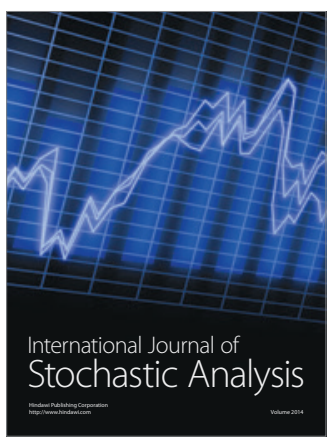

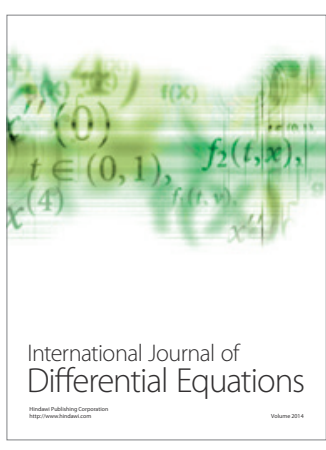
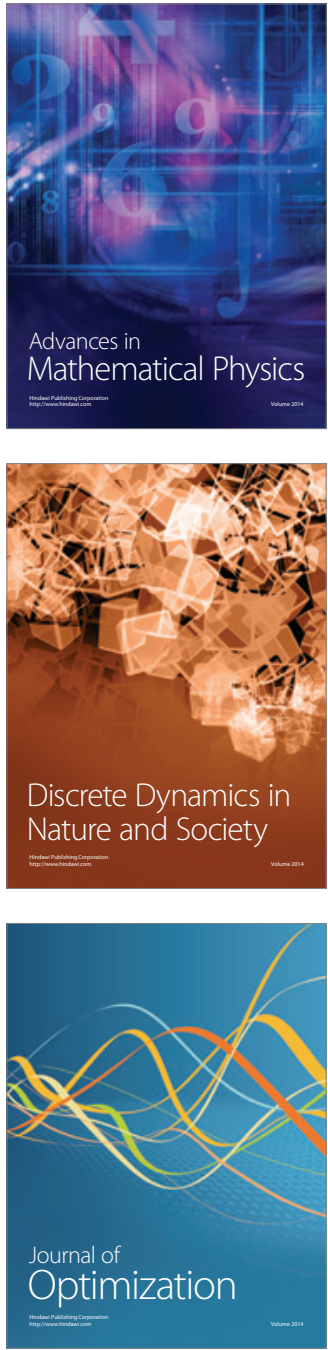